\title{
Study of Correlation of Preoperative Fine Needle Aspiration Cytology with Histopathological Examination in Thyroid Swellings
}

\author{
${ }^{1}$ Sri Lekha Bodepudi, ${ }^{2}$ KASSN Kalyan, ${ }^{3}$ Ramarao Peddi
}

\begin{abstract}
Introduction: Though fine needle aspiration cytology (FNAC) for thyroid is acclaimed as a gold standard before going for intervention, there are some limitations with respect to tissue availability, technique, skill of performer, etc. So, a crosssectional study was conducted to determine the accuracy of FNAC in diagnosis of thyroid swelling and to assess the correlation between preoperative cytopathological diagnosis and postoperative histopathological diagnosis.
\end{abstract}

Materials and methods: Data collected from records in the time period of 3 years, i.e., from August 2012 to August 2015, were evaluated and analyzed.

Results: A total number of 93 cases were included in the study of which majority were females with frequency of $84.94 \%$, most of the incidence of thyroid swellings occur in the age group of 40 to 50 years. Overall mismatch between histopathological examination and FNAC was 43 out of 93 and of which 7 were major and 36 were minor mismatch. Sensitivity of patients having malignancy with positive FNAC is $82.35 \%$ from the study and specificity of patients with nonmalignant thyroid disease and positive cytology is $95.18 \%$.

Conclusion: It was observed that FNAC is a reliable, safe, and accurate method as a first line of evaluation in thyroid swelling before the surgery. Fine needle aspiration cytology is more sensitive in detecting thyroid gland malignancy but still correlation is always necessary and therefore, histopathological analysis still remains essential for final diagnosis.

Keywords: Fine needle aspiration cytology, Follicular adenoma, Histopathological examination, Malignancy, Thyroid swelling.

How to cite this article: Bodepudi SL, Kalyan KASSN, Peddi R. Study of Correlation of Preoperative Fine Needle Aspiration Cytology with Histopathological Examination in Thyroid Swellings. Int J Phonosurg Laryngol 2017;7(1):16-19.

Source of support: Nil

Conflict of interest: None

${ }^{1}$ Student, ${ }^{2}$ Junior Resident, ${ }^{3}$ Associate Professor

${ }^{1-3}$ Department of General Surgery, Dr. Pinnamaneni Siddhartha Institute of Medical Sciences \& Research Foundation Gannavaram, Andhra Pradesh, India

Corresponding Author: Sri Lekha Bodepudi, Student Department of General Surgery, Dr. Pinnamaneni Siddhartha Institute of Medical Sciences \& Research Foundation Gannavaram, Andhra Pradesh, India, Phone: +918019195123 e-mail: lekhabodepudi92@gmail.com

\section{INTRODUCTION}

It is estimated that 4 to $7 \%$ adults have palpable enlargement of thyroid and 10 times more patients have nodules which are not clinically palpable and fewer than $5 \%$ among them are actually malignant. ${ }^{1}$

Among multitude of diagnostic tests like ultrasound of neck, fine needle aspiration cytology (FNAC) and many more are available to evaluate a case of goiter. Final diagnosis always requires morphological examination of lesions for which FNAC and histopathological examination (HPE) becomes mandatory tests. ${ }^{2}$

Though FNAC as a method was first published by Leyden in $1883,{ }^{3}$ the application for thyroid lesions was first reported by Martin and Ellis in 1930. ${ }^{4}$ Practice guidelines set forth by American Thyroid Association and National Comprehensive Cancer Network state that FNAC should be used as initial diagnostic test because of its superior diagnostic reliability and cost-effectiveness. ${ }^{3}$

Fine needle aspiration cytology is considered the gold standard in the evaluation of thyroid swelling. It is a simple, cost-effective, readily repeated, and quick to perform procedure in the outpatient department, with excellent patient compliance. Important factor for satisfactory test includes representative specimen from the goiter and an experienced cytologist to interpret the findings. ${ }^{2}$

Fine needle aspiration cytology, however, is not without limitations related to specimen adequacy, sampling techniques, skill of performing the aspiration, interpretation of the aspirate, and overlapping cytological features between benign and malignant follicular neoplasm and also in the detection of some papillary carcinomas because of associated thyroid pathology including multinodular goiter (MNG), thyrotoxicosis, and marked cystic changes.

Here arises the need for HPE, as it is considered the final diagnostic test. Thus, even if nonsurgical and noninvasive techniques can provide a diagnosis, the ultimate answer rests in the HPE of the excised thyroid tissue. This also raises the question of how much corroborative is FNAC and HPE. ${ }^{3}$

This study is carried out with the objective of comparing the findings of the two tests, namely FNAC 
and HPE, and suggestions for the future. It is to compare the diagnostic accuracy of preoperative FNAC in thyroid swellings with postoperative HPE results, especially in diagnosing malignancy.

\section{MATERIALS AND METHODS}

This is a retrospective cross-sectional study where the data were collected from patient records from the Department of General Surgery in a tertiary care hospital.

- This is a cross-sectional study design.

- Period of study is from August 2012 to August 2015 and 93 patients were included in the study.

- All the patients included had FNAC reports of thyroid swellings which were done on outpatient department basis and having postoperative histopathological reports from thyroidectomy specimens postsurgery. Both the reports were evaluated and analyzed.

\section{Inclusion Criteria}

Patients with thyroid swellings and euthyroid having FNAC report who underwent thyroidectomy.

\section{RESULTS}

In this series of 93 thyroid swellings, females were higher in frequency $(\mathrm{n}=79 ; 84.94 \%)$ than males $(\mathrm{n}=14 ; 15.05 \%)$ (Table 1).

Patients were grouped in age groups of 0 to $10 ; 10$ to $20 ; 20$ to $30 ; 30$ to $40 ; 40$ to $50 ; 50$ to $60 ; 60$ to 70 ; and 70 to 80 years and patients in each age group were 1.07, 7.5, $20.4,23.6,26.8,15.05,4.3$, and $1.07 \%$ respectively. Most of the patients were in the age group of 40 to 50 years [25 (26.88\%)] followed by 30 to 40 years [22 (23.65\%)] (Table 2).

On FNAC, 53 cases (56.98\%) had non-neoplastic and 40 cases $(43.01 \%)$ had neoplastic disease (Table 3$)$.

Table 1: Sex distribution

\begin{tabular}{ll}
\hline Males & $14(15.05 \%)$ \\
Females & $79(84.94 \%)$ \\
\hline Total & 93 \\
\hline
\end{tabular}

Table 2: Age distribution

\begin{tabular}{ll}
\hline Age group (years) & Number \\
\hline $0-10$ & $1(1.07 \%)$ \\
$10-20$ & $7(7.5 \%)$ \\
$20-30$ & $19(20.4 \%)$ \\
$30-40$ & $22(23.6 \%)$ \\
$40-50$ & $25(26.8 \%)$ \\
$50-60$ & $14(15.05 \%)$ \\
$60-70$ & $4(4.3 \%)$ \\
$70-80$ & $1(1.07 \%)$ \\
\hline Total & 93 \\
\hline
\end{tabular}

Table 3: Fine needle aspiration cytology of all cases

\begin{tabular}{ll}
\hline Non-neoplastic & 53 \\
Neoplastic & 40 \\
\hline Total & 93 \\
\hline
\end{tabular}

Table 4: Fine needle aspiration cytology results of non-neoplastic swellings

\begin{tabular}{ll}
\hline Multinodular goiter & 20 \\
Adenomatous goiter & 10 \\
Nodular goiter & 9 \\
Colloid goiter & 4 \\
Hashimoto's thyroiditis & 8 \\
Ectopic thyroid & 1 \\
Graves' disease & 1 \\
\hline Total & 53 \\
\hline
\end{tabular}

Among the non-neoplastic swellings, MNG was most common with 20 cases $(37.73 \%)$, followed by adenomatous goiter which were 10 cases $(18.86 \%)$ and nodular goiter which were 9 cases (16.98\%) (Table 4).

Among the neoplastic thyroid swelling, follicular neoplasm was commonest with 22 cases (55\%) followed by papillary carcinoma with 17 cases $(42.5 \%)$ (Table 5$)$.

After postoperative HPE, 79 cases $(84.94 \%)$ were found nonmalignant and 14 cases $(15.05 \%)$ were found malignant (Table 6).

Among 79 cases of nonmalignant thyroid swelling were colloid goiter with 30 cases $(37.97 \%)$, followed by follicular adenoma 15 cases $(18.98 \%)$, nodular goiter 14 cases $(17.72 \%)$ adenomatous goiter with 9 cases (11.39\%), MNG with 5 cases (6.32\%) (Table 7).

Table 5: Fine needle aspiration cytology results of neoplastic swellings

\begin{tabular}{ll}
\hline Follicular neoplasm & 22 \\
Papillary carcinoma & 17 \\
Medullary carcinoma & 01 \\
\hline Total & 40 \\
\hline
\end{tabular}

Table 6: Histopathology reports

\begin{tabular}{ll}
\hline Malignant & 14 \\
Nonmalignant & 79 \\
\hline Total & 93 \\
\hline
\end{tabular}

Table 7: Histopathology reports of nonmalignant swellings

\begin{tabular}{ll}
\hline Colloid goiter & 30 \\
Follicular adenoma & 15 \\
Nodular goiter & 14 \\
Adenomatous goiter & 9 \\
Multinodular goiter & 5 \\
Hashimoto's thyroiditis & 3 \\
Graves' disease & 1 \\
Thyroglossal cyst & 2 \\
\hline Total & 79 \\
\hline
\end{tabular}


Table 8: Histopathology reports of malignant swellings

\begin{tabular}{ll}
\hline Papillary carcinoma & 13 \\
Medullary carcinoma & 01 \\
\hline Total & 14 \\
\hline
\end{tabular}

Table 9: Mismatch between FNAC and histopathology

\begin{tabular}{ll}
\hline Major & 7 \\
Minor & 36 \\
\hline Total & 43 \\
\hline
\end{tabular}

Among the malignant thyroid swelling, papillary carcinoma was commonest with 13 cases (92.85\%) and medullary carcinoma with 1 case (Table 8).

On comparing the findings of FNAC and postoperative histopathology examination, we see overall mismatch is 43 out of 93 cases $(46.23 \%)$ of which $7(7.52 \%)$ were major mismatch and 36 (38.71\%) were minor mismatch (Table 9), considering mismatch of benign FNAC/histopathological report with malignant FNAC/histopathological report as major mismatch (either missing malignancy in FNAC or diagnosis as benign through histopathology) and mismatch of benign FNAC/histopathological report with other benign FNAC/histopathological report as minor mismatch (benign diagnostic mismatch).

Among major mismatch group, commonest mismatch was FNAC revealing follicular neoplasm and histopathology as papillary carcinoma in two cases $(28.57 \%)$ and FNAC as papillary carcinoma and histopathology as nodular goiter in two cases (28.57\%), FNAC as papillary carcinoma and histopathology as colloid goiter in one case (14.28\%) (Table 10$)$.

Among minor mismatch group, commonest mismatch was FNAC as follicular neoplasm and histopathology as colloid goiter in eight cases (22.22\%) followed by FNAC as MNG and histopathology as colloid goiter in seven cases (19.44\%), FNAC as follicular neoplasm and histopathology as MNG in five cases (13.88\%) (Table 11).

Overall major mismatch was $7.52 \%$ out of which four cases of FNAC revealing malignancies were ruled out as malignancies with histopathology report. So sensitivity shows the portion of the patients having malignant thyroid disease and positive cytological diagnosis on

Table 10: Major mismatch

\begin{tabular}{lll}
\hline FNAC & Histopathology & Number \\
\hline Papillary carcinoma & Nodular goiter & 2 \\
Papillary carcinoma & Colloid goiter & 1 \\
Papillary carcinoma & Adenomatous goiter & 1 \\
Follicular neoplasm & Papillary carcinoma & 2 \\
Multinodular goiter & Papillary carcinoma & 1 \\
\hline Total & & 7 \\
\hline
\end{tabular}

Table 11: Minor mismatch

\begin{tabular}{lll}
\hline FNAC & Histopathology & Number \\
\hline Follicular neoplasm & Colloid goiter & 8 \\
Multinodular goiter & Colloid goiter & 7 \\
Hashimoto's thyroiditis & Colloid goiter & 4 \\
Adenomatous goiter & Colloid goiter & 4 \\
Hashimoto's thyroiditis & Adenomatous goiter & 1 \\
Hashimoto's thyroiditis & Nodular goiter & 1 \\
Ectopic thyroid & Thyroglossal cyst & 1 \\
Nodular goiter & Colloid goiter & 3 \\
Multinodular goiter & Adenomatous goiter & 1 \\
Follicular neoplasm & Multinodular goiter & 5 \\
Adenomatous goiter & Multinodular goiter & 1 \\
\hline Total & & 36 \\
\hline
\end{tabular}

FNAC, which is found to be $82.35 \%$. Specificity shows the portion of the patients with nonmalignant thyroid disease and positive cytological diagnosis, which was found to be 95.18\%: Positive predictive value (PPV) is the probability of having malignant thyroid disease following a positive FNAC finding and is found to be $77.77 \%$ here. Negative predictive value (NPV) is the probability of not having malignant thyroid disease following a negative FNAC finding and is found to be $96.34 \%$.

\section{DISCUSSION}

Fine needle aspiration cytology contributes significantly to the preoperative investigation in patients with thyroid swelling, but despite its well-recognized value, there are limitations to the technique. The reported pitfalls are those related to specimen adequacy, ${ }^{4,5}$ sampling techniques, the skill of the operator performing the aspirations, the experience of the cytopathologist interpreting the aspirate, and overlapping cytological features between benign and malignant follicular neoplasms and inadequate, indeterminate FNAC. One major limitation of thyroid cytology is its inability to distinguish between follicular adenoma from follicular carcinoma. ${ }^{5-7}$ This diagnosis requires detailed histological examination for vascular or capsular invasion and cannot be reliably made on routine FNAC specimens. ${ }^{8,9}$ Hence, follicular neoplasm (lesion) is given as diagnosis in FNAC. In the present study of 93 cases, majority of cases were seen in the age group of 40 to 50 years $(26.88 \%)$. Female preponderance $(84.94 \%)$ was compared with male preponderance $(15.05 \%)$. The youngest patient of this series was a boy of 8 years with ectopic thyroid and the oldest patient was a female of 72 years, a case of papillary carcinoma.

In this study, FNAC showed $43.01 \%$ neoplastic cases. Among neoplastic thyroid swellings, follicular neoplasm was common with 22 cases (55\%) followed by papillary carcinoma with 17 cases (42.5\%). Carcinoma of the thyroid is the most common malignancy of endocrine 
system comprising 0.6 and $1.6 \%$ of all cases of malignant neoplasm in men and women respectively.

In this study, among non-neoplastic thyroid swelling $(56.98 \%)$, MNG was common which was $37.73 \%$ and adenomatous goiter with $18.86 \%$. The study reported by Shafiqul et al ${ }^{10}$ showed $78 \%$ non-neoplastic and $22 \%$ neoplastic lesions (papillary carcinoma, $15.56 \%$ and follicular lesion 3.3\%).

In this study, histopathologically $84.94 \%$ were nonmalignant and $15.05 \%$ malignant, and among nonmalignant colloid goiter was commonest with 30 cases (37.97\%; $32.25 \%$ of total cases) followed by nodular goiter with 14 cases (17.72\%). Among malignant swellings, commonest was papillary carcinoma with 13 cases $(92.85 \% ; 13.97 \%$ of total cases) followed by papillary carcinoma with 1 case $(7.14 \%)$.

While comparing FNAC with HPE, overall mismatch is 43 out of 93 cases of which 7 were major mismatch and 36 were minor mismatch. In major mismatch group, commonest mismatch was with pre-op FNAC revealing follicular neoplasm and biopsy as papillary carcinoma in two cases and FNAC as papillary carcinoma and biopsy nodular goiter in two cases, FNAC as MNG and cytology as papillary carcinoma in one case, FNAC as papillary carcinoma and cytology as colloid goiter and adenomatous goiter in one case, each nonmalignant (significant for planning management). Lewis et $\mathrm{al}^{11}$ reported that 15 to $30 \%$ of FNAs diagnosed as follicular neoplasm were actually carcinomas; remaining 70 to $85 \%$ were benign.

Cytohistological correlation was present in $92.47 \%$ of cases in this study. Choudhury et $\mathrm{al}^{12}$ reported cytological correlation in $80 \%$ of cases. Pandey et $\mathrm{al}^{13}$ also reported cytohistological concordance in $80.28 \%$ of the cases. In the present study, we found sensitivity by FNAC to be $82.35 \%$, the specificity was $95.18 \%$, the PPV was $77.77 \%$, and NPV was $96.34 \%$. Several international studies have documented the sensitivity of FNAC in thyroid nodules to range from 52 to $98 \%$. Similarly, the international normal range is for specificity 72 to $100 \%$ and for PPV 50 to $90 \%$. The NPV derived from this audit was $73.1 \%$. In a report of 19 studies and 20 series by Lewis et al, ${ }^{11}$ the reported and recalculated mean for NPV was 84 and 93\%. So, present study is fairly comparable to other studies.

\section{CONCLUSION}

Fine needle aspiration cytology is a simple, safe, and cost-effective diagnostic modality in the investigation of thyroid disease with high specificity and accuracy. We concluded that FNAC diagnosis of malignancy is highly significant. A benign FNAC diagnosis should be viewed with caution as false-negative results do occur and these patients should be followed up and any clinical suspicion of malignancy even in the presence of benign FNAC requires surgery. So, final diagnosis and treatment pattern should be based upon histopathology.

\section{REFERENCES}

1. Esmaili HA, Taghipour H. Fine needle aspiration in the diagnosis of thyroid diseases: an appraisal in our institution. ISRN Pathol 2012 Aug;2012:1-4.

2. Gupta M, Gupta S, Gupta VB. Correlation of fine needle aspiration cytology with histopathology in the diagnosis of solitary thyroid nodule. J Thyroid Res 2010 Feb;2010:1-5.

3. Das D, Sarma MC, Sharma A, Datta TK, Lahiri SK. A comparative study between fine needle aspiration cytology and histopathological examination in the diagnosis of neoplastic and nonneoplastic lesions of the thyroid gland. Indian J Prev Soc Med 2012 Jan-Mar;43(1):72-75.

4. Löwhagen T, Willems J, Lundell G, Sundblad R, Granberg P. Aspiration biopsy cytology in diagnosis of thyroid cancer. World J Surg 1981 Jan;5(1):61-73.

5. Al-Sayer HM, Krukowski ZH, Williams VM, Matheson NA. Fine needle aspiration cytology in isolated thyroid swellings: a prospective two year evaluation. Br Med J (Clin Res Ed) 1985 May;290(6480):1490-1492.

6. Anderson JB, Webb AJ. Fine-needle aspiration biopsy and the diagnosis of thyroid cancer. Br J Surg 1987 Apr;74(4): 292-296.

7. Leonard N, Melcher DH. To operate or not to operate? The value of fine needle aspiration cytology in the assessment of thyroid swellings. J Clin Pathol 1997 Nov;50(11):941-943.

8. Gardner HA, Ducatman BS, Wang HH. Predictive value of fine-needle aspiration of the thyroid in the classification of follicular lesions. Cancer 1993 Apr;71(8):2598-2603.

9. Miller JM, Kini SR, Hamburger JI. The diagnosis of malignant follicular neoplasms of the thyroid by needle biopsy. Cancer 1985 Jun;55(12):2812-2817.

10. Shafiqul IMd, Siddiquee BH, Akhtar N, Salam KS, Aktaruzzaman M. Comparative study of FNAC and histopathology in the diagnosis of thyroid swelling. Bangladesh J Otorhinolaryngol 2010 Apr;16(1):35-43.

11. Lewis CM, Chang KP, Pitman M, Faquin WC, Randolph GW. Thyroid fine-needle aspiration biopsy: variability in reporting. Thyroid 2009 Jul;19(7):717-723.

12. Choudhury M, Singh S, Agarwal S. Diagnostic utility of ki67 and p53 immunostaining on solitary thyroid nodule - a cytohistological and radionuclide scintigraphic study. Indian J Pathol Microbiol 2011 Jul-Sep;54(3):472-475.

13. Pandey P, Dixit A, Mahajan NC. Fine-needle aspiration of the thyroid: a cytohistologic correlation with critical evaluation of discordant cases. Thyroid Res Pract 2012 May;9(2):32-39. 